\title{
Positronium in Metal-Oxide Powders Studied with Age. The Age-Momentum Correlation Technique
}

\author{
B. Van Waeyenberge and Ch. Dauwe \\ NUMAT, Department of Subatomic and Radiation Physics, Ghent University \\ Proeftuinstraat 86, 9000 Gent, Belgium
}

\begin{abstract}
For the first time positronium is investigated in fine powders of $\mathrm{MgO}$ and $\mathrm{Al}_{2} \mathrm{O}_{3}$ using age-momentum correlation technique based on a relativistic positron beam. The application of this technique for investigating the interaction of positronium with the grain surfaces is discussed and compared with other techniques. The previously reported interaction of the positronium with paramagnetic centres is further studied. A qualitative interpretation of the spectra is given. In the $\mathrm{Al}_{2} \mathrm{O}_{3}$ samples we found some unexpected behaviour of the conversion quenching of ortho-positronium at irradiation induced paramagnetic surface defect.
\end{abstract}

PACS numbers: 36.10.Dr, 41.75.Fr, 78.70.Bj

\section{Introduction}

The interaction of positrons with matter has been studied extensively for the last 5 decades. Most of the materials studied were metals and one can say that the interaction of the positron with metals is well understood right now. In simple systems, both lifetime and Doppler broadening parameters can be predicted by theory with reasonable accuracy. In contrary positron annihilation in insulators is less understood. However, during the last 10 years there has been an increased interest in the use of positron annihilation techniques for the study of insulators, more specific polymers. Interpretation of data is not unique and is a subject to a lot of discussions.

The low electron density regions in these insulators allow the formation of positronium (Ps). This is a hydrogen-like bound state of an electron and its antiparticle, a positron. The ground state consists of a triplet of spin-1 states, ortho-positronium (o-Ps) and a singlet spin-0 state, para-positronium ( $p$-Ps). In 
vacuum they will decay into 3 and 2 gamma quanta, respectively, and with a mean lifetime of $142 \mathrm{~ns}$ and $125 \mathrm{ps}$, respectively. Positronium formation and decay have been investigated for a wide range of solids, liquids, and gases. Nevertheless, the behaviour of this neutral "atom" is not completely understood in most materials. The positronium formation, trapping, and thermalisation are still key issues which strongly influence interpretation of positron data.

Fine powders of metal oxides like $\mathrm{MgO}, \mathrm{Al}_{2} \mathrm{O}_{3}$, and $\mathrm{SiO}_{2}$ are known for their high positronium formation probabilities. Because of their low density the ortho-positronium lives much longer than the unbound positrons in the grains. This long lifetime makes it possible to discriminate between positron and positronium annihilation and makes these powders interesting materials to study the positronium physics.

When an energetic positron is injected into a fine powdered material, it will lose this energy very fast when it passes through the grains until it is stopped at near thermal energy. In this process positronium may be formed inside a grain and diffuse to the grain surface. If Ps work function is negative, it will be emitted from the surface into free space between the grains. This mechanism is believed to be responsible for the emission of $\mathrm{Ps}$ from $\mathrm{SiO}_{2}$. Alternatively, if $\mathrm{Ps}$ is not formed inside the grain, the positron itself may diffuse to the surface and escape in the form of Ps by picking up an electron from the surface. This process must be responsible for the emission of Ps from metal surfaces where Ps cannot exist in the bulk. There are some indications that this is also the case for the emission from $\mathrm{Al}_{2} \mathrm{O}_{3}$ and $\mathrm{MgO}$ surfaces. Experiments have shown that the ortho-positronium is emitted from the surface of the grains with a few $\mathrm{eV}$ of kinetic energy and will lose this energy by numerous interactions with the walls of the grains. Meanwhile the $o$-Ps will annihilate through self-annihilation as well as collisional quenching of the positron with electrons of the grain surface, called pick-off quenching.

Because the positronium mass is very low, thermalisation by collisions with the grains is very slow. When we consider classical elastic scattering, the energy loss for one collision is

$$
\Delta E_{\mathrm{Ps}}=-4 \frac{m_{\mathrm{Ps}}}{M}\left(E_{\mathrm{Ps}}-E_{\mathrm{th}}\right)
$$

with $m_{\mathrm{Ps}}$ - the positronium mass, $M$ - the effective mass of the recoiling atoms and $E_{\mathrm{th}}$ - the mean thermal energy. The Ps kinetic energy, as it asymptotically approaches thermal energy $E_{\mathrm{th}}$, is given by

$$
E(t) / E_{\text {th }}=\operatorname{coth}^{2}(\beta+\Lambda t),
$$

where $\beta$ relates to the initial energy, $E_{0}=E_{\mathrm{th}} \operatorname{coth}^{2}(\beta)$ and $\Lambda$ - the thermalisation rate. This thermalisation relates the mean free path $\delta$ of the positronium as

$$
A=4 \frac{p_{\text {th }}}{\delta M}
$$

with $p_{\text {th }}$ - the positronium momentum at thermal energy. This thermalisation 
model was first deduced by Sauder [1] for the thermalisation in noble gasses and later also applied to Ps in powders by others [2-5].

The experiment of Chang et al. [2] showed that thermalisation of $o$-Ps in a low-density silica aerogel takes longer than $60 \mathrm{~ns}$ and follows this elastic thermalisation law. Only a significant deviation was found for the first data point representing the first $3 \mathrm{~ns}$, indicating inelastic thermalisation for energies above $0.25 \mathrm{eV}$. Later, a more accurate experiment [6] gave no sign of inelastic collisions up to the zero age energy of $0.6 \mathrm{eV}$. The model was also used successfully by several authors for explaining data in low-density gasses $[4,5]$.

If paramagnetic centres are available, the $o$-Ps will be quenched by $o$-Ps to $p$-Ps conversion. Spin exchange interaction with this paramagnetic centre converts the long living $o$-Ps into the short living $p$-Ps. When powders are cooled to low temperature (below $150 \mathrm{~K}$ ) with an $\mathrm{e}^{+}$source nearby, the grain surface accumulates such defects. These positron-irradiation-induced defects were first observed in $\mathrm{Al}_{2} \mathrm{O}_{3}$ powder [7]. There was a clear change of the tree-quantum yield in function of the radiation time. Later a more systematic study with tree-quantum yield and positron lifetime experiments revealed that this effect was due to $o-p$ conversion on defects [8]. A similar positron-irradiation time dependence of the $o$-Ps lifetime was observed in $\mathrm{MgO}$ [9] and later by Saito et al. [10] at 1000 times higher doses in $\mathrm{SiO}_{2}$. More recently it has been shown that paramagnetic defects in $\mathrm{Al}_{2} \mathrm{O}_{3}$ and $\mathrm{SiO}_{2}$ at low temperatures could also be created by irradiation with UV light [11].

\section{Experimental technique}

Thermalisation of positronium has been measured both indirectly as directly. Indirectly this thermalisation is observed as non-exponential decay of the $o$-Ps $[4,9]$. The collisional quenching rate will change as a function of the velocity of the positronium. Performing these experiments is relatively simple but data interpretation is complicated because a spectrum also contains a strong contribution of the annihilation of free positron not forming positronium. This component with a mean lifetime of typical $300 \mathrm{ps}$ masks the strongest thermalisation effects of the $o$-Ps components.

More directly, the time dependence of the kinetic energy can be measured with Age-MOmentum Correlation (AMOC) technique. This technique measures the positron lifetime simultaneously with the momentum, either by Doppler broadening or angular correlation measurement. Because both annihilation photons need to be detected, data acquisition is slower and therefore stronger positron sources or intense positron beams are needed.

Despite the high momentum resolution of the time resolved angular correlation experiments, they have unpractical low count rates because the momentum information is derived from the small angular deviation of the collinearity of the two gamma decay quanta. Takada et al. [6] reports a count rate of $0.027 \mathrm{c} / \mathrm{s}$ and a data collection time of $2000 \mathrm{~h}$ with a source based set-up. 
The Doppler broadening technique using a high resolution Ge-detector has a lower momentum resolution in favour of higher count rates. Several groups report a successful use of this technique for studying positronium thermalisation. Chang et al. [2] reported the first measurements in silicon oxide powder with a source base set-up. Gidley et al. [5] used this technique to study positronium thermalisation in gases.

To measure the momentum of the Ps it should decay through 2-gamma decay. Because the natural decay mode of $o$-Ps is 3-gamma decay, one should present some conversion to detect it in the AMOC spectrometer. This 2-gamma signal will also have a contribution of pick-off quenching which carries mainly the momentum of grain surface electrons. The conversion to 2-gamma decay of $o$-Ps can be obtained in two ways. When a magnetic field is applied, the two states (o-Ps and $p$-Ps) mix up and a part of the long component will annihilate into 2 gammas. Otherwise conversion can be obtained by introducing paramagnetic centres to convert some of the $o$-Ps to $p$-Ps which will subsequently annihilate by 2-gamma decay. Because of the low energy difference between both states $(0.84 \mathrm{meV})$ this spin exchange interaction is believed to conserve the original o-Ps momentum.

The AMOC experiments presented here are performed at the relativistic positron beam facility in Stuttgart. A pelletron is used to accelerate moderated positrons to $3 \mathrm{MeV}$. In the high voltage terminal positrons from a $50 \mathrm{mCi}{ }^{22} \mathrm{Na}$ source are moderated by a single crystal tungsten moderator (see Fig. 1). The monoenergetic low energy positrons emitted are accelerated by the electric field to form a monoenergetic beam of positrons. After transition through a thin $(3 \mathrm{~mm})$ plastic scintillator coupled to photo multiplier, the positrons can be injected into any sample. The signal from the scintillator delivers the start reference signal for the determination of the lifetime of the positrons. Around the sample two detectors are placed opposite to each other to detect the annihilation quanta. $\mathrm{A}$ fast $\mathrm{BaF}_{2}$ scintillation detector is used to one of the photons to deliver the stop reference signal for the lifetime measurement. Coincidently a high purity Ge-detector is used to measure the Doppler broadening of the other photon. This lifetime spectrometer using the positron beam has a number of significant advantages over the standard source sandwich based systems. The detection efficiency of the start detector is nearly $100 \%$. This high efficiency gives a high peak to background ratio, typically three orders of magnitude better than the standard lifetime technique where the efficiency is of the order of $10 \%$. Combined with the much higher count rate it allows for fast acquisition of accurate lifetime spectra.

In other AMOC set-ups the start signal is also derived from the transition of positrons through a thin plastic scintillator before entering the sample. Because of the continuous energy spectrum delivered by a $\beta^{+}$-emitter, a lot of positrons will annihilate in the scintillator and a big fraction of redundant signals will limit the source strength to be used. The best resolution reported is typically $2-3 \mathrm{~ns}$ [2]. 


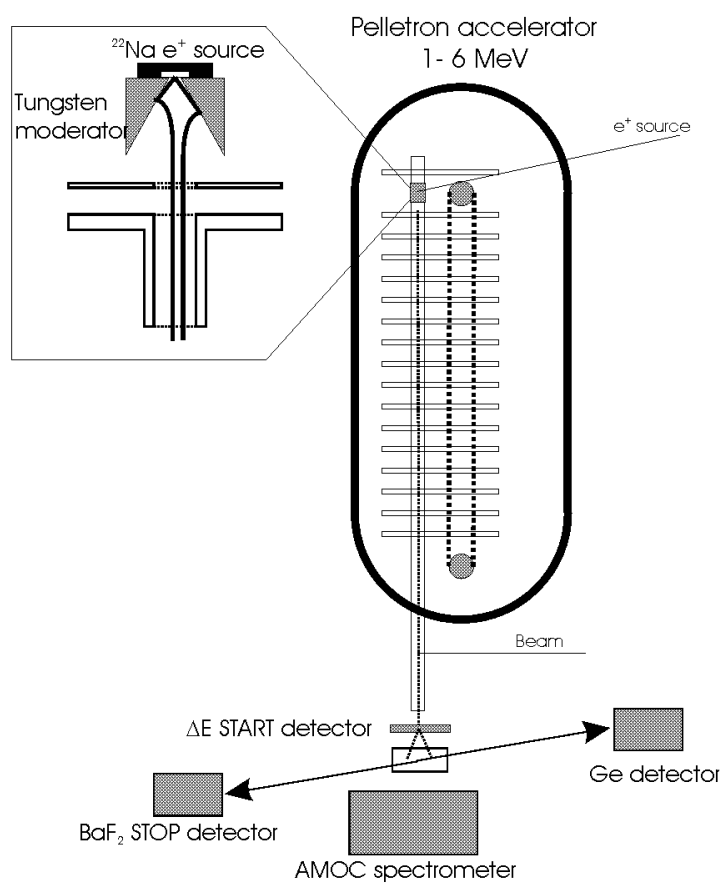

Fig. 1. Schematic of the relativistic positron beam and the AMOC set-up.

When a slightly thicker $(5 \mathrm{~mm})$ start scintillator is used at the beam time resolutions down to 200 ps FWHM can be achieved. The mean disadvantages of this beam is the need for relatively bigger samples because more the positrons enter the sample with more than $2.5 \mathrm{MeV}$ which corresponds to a range of about $20 \mathrm{~mm}$ in low density powders pellets $\left(\rho \cong 0.7 \mathrm{~g} / \mathrm{cm}^{3}\right)$.

The pelletron was operated at $3 \mathrm{MV}$ and delivered $400000 \mathrm{e}^{+} / \mathrm{s}$. Because of this high number of incoming positrons care had to be taken to reject all cases where two positrons are injected in the sample within the correlation time window (multiple impact rejection). Without this a background in the lifetime spectrum would appear even worse than with a conventional source-based lifetime spectrometer. Our data acquisition system allowed for the recording of both single lifetime and energy spectrum at full count rate together with the correlated AMOC data without duplicating the ADC's. In this way we can acquire simultaneously single spectra and monitor the stability of the electronics.

The count rate in the lifetime spectrum was $17000 \mathrm{c} / \mathrm{s}, 14000 \mathrm{c} / \mathrm{s}$ in the energy spectrum and $200 \mathrm{c} / \mathrm{s}$ in the triple coincidence AMOC spectrum. The time resolution was $750 \mathrm{ps}$ FWHM and lifetime spectra were recorded with a $1000 \mathrm{~ns}$ time window. The energy resolution of the Ge-detector was $1.4 \mathrm{keV}$ and was monitored by the $497 \mathrm{keV}$ line of the ${ }^{103} \mathrm{Ru}$ isotope placed near the detector. 
Samples of fine powders of $\mathrm{MgO}$ an $\mathrm{Al}_{2} \mathrm{O}_{3}$ with mean grain diameters of $40 \mu \mathrm{m}$ and $50 \mathrm{~nm}$, respectively, were prepared. They were pressed into pellets with a density of $0.88 \mathrm{~g} / \mathrm{cm}^{3}$ for $\mathrm{MgO}$ and $0.65 \mathrm{~g} / \mathrm{cm}^{3}$ for $\mathrm{Al}_{2} \mathrm{O}_{3}$.

One of the samples of each material was mixed with $200 \mu \mathrm{Ci}$ of a short living $\beta$ emitter, ${ }^{32} \mathrm{P}$ under the form of $\mathrm{KHPO}_{2}$. This source was selected to increase the number of radiation induced surface defects at low temperatures without adding perturbing gamma radiation during the experiment.

The samples were mounted on a cold finger of a closed cycle helium refrigerator in a vacuum chamber allowing measurements in a temperature range of $20-450 \mathrm{~K}$. The vacuum pressure was $5 \times 10^{-7}$ mbar at room temperature and $1 \times 10^{-8} \mathrm{mbar}$ at low temperature. Before the cooling, each sample was degassed under vacuum at $450 \mathrm{~K}$ for about $5 \mathrm{~h}$.

The non-irradiated $\mathrm{MgO}$ sample was additionally measured under an $\mathrm{O}_{2}$ gas pressure of $200 \mathrm{mbar}$. This gas diffuses into free space of the sample and conversion quenches the $o$-Ps component. Measurement time varied from 24 hours to 5 days.

\section{Results and discussion}

Contrary to what was expected from previous lifetime experiments [9], low temperature experiments on the pure $\mathrm{MgO}$ sample at $25 \mathrm{~K}$ showed almost no conversion quenching. Because the long living o-Ps component decayed almost exclusively by 3-gamma decay, the AMOC spectra contained almost no counts for the longer age. In the lifetime spectrum only very slow quenching was observed. Although the energy deposition rate in the sample by the positron beam is equivalent to that of a $100 \mu \mathrm{Ci}^{22} \mathrm{Na}$ source in a sandwich geometry, this energy is spread out over a much higher volume in the case of the beam. Even in the MgO sample with extra $\beta$ source, no significant conversion quenching was observed. This source gives a 10 times higher dose than the beam, homogeneous over the whole sample. We attribute this difference, as compared with the previous reported results on $\mathrm{MgO}$, to the different origin of the sample. A different production, and purification technique might alter the condition of the grain surfaces.

When the $\mathrm{MgO}$ sample was brought under an oxygen atmosphere, a high level of conversion quenching for longer age was observed. The $S$-parameter as a function of positron age is shown in Fig. 2. A detailed analysis of the data with different models is reported elsewhere [12]. The results are in agreement with the elastic thermalisation model, however not conclusive because the high oxygen pressure makes thermalisation fast so its typical AMOC signal is mixing with the shorter positron components.

In contrast the $\mathrm{Al}_{2} \mathrm{O}_{3}$ samples show a clear build-up of paramagnetic defect when cooled to $25 \mathrm{~K}$. The spectra with and without $\beta$ source are similar except for the higher conversion rate in the former. The $S$-parameter versus positron age is shown in Fig. 3. This parameter is calculated as the fraction of the counts in the 


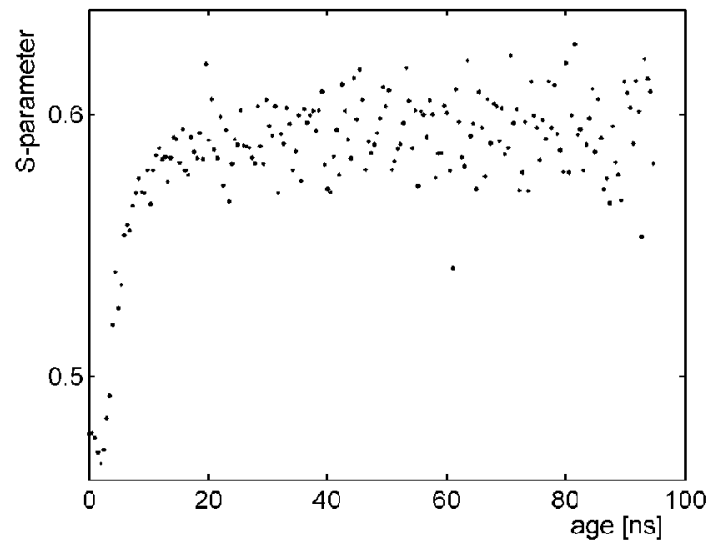

Fig. 2. Age depend $S$-parameter of $\mathrm{MgO}$ sample measured in a $200 \mathrm{mbar} \mathrm{O}_{2}$ atmosphere.

central range to the total of the photo peak. The range is selected to have a nominal value of $S=0.5$. The structure in the first 20 ns of the spectrum can completely be attributed to the short positron components. They carry the typical higher momentum distribution of the electrons of the grains where the annihilation takes place and so this contribution has a low $S$-parameter. The positron components with typical lifetimes of 200-300 ps have completely died away after 20 ns. We see that then the $S$-parameter has a high value indicating low annihilation momentum, which indicates the ortho to para conversion.

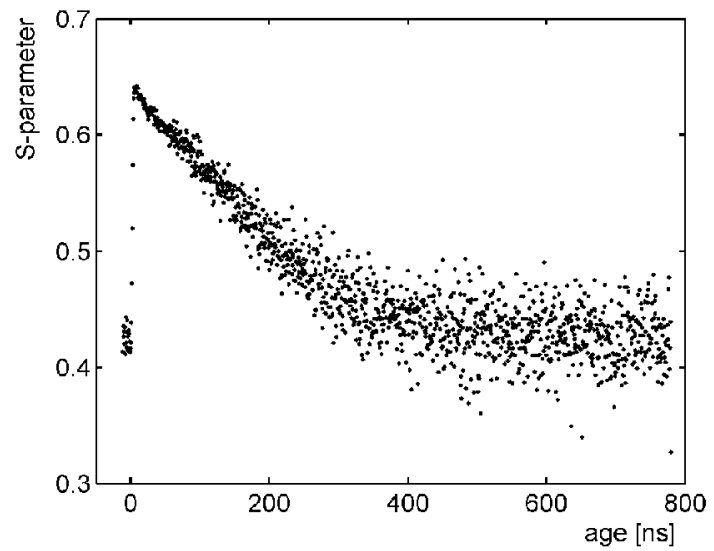

Fig. 3. Age depend $S$-parameter of $\mathrm{Al}_{2} \mathrm{O}_{3}$ sample at $25 \mathrm{~K}$ after 8 days of irradiation by the ${ }^{32} \mathrm{P}$ source.

At much longer age a slow decrease in $S(t)$ can be observed. This is the opposite effect of a decrease in the mean positronium momentum by thermalisation. 
To our knowledge this effect has never been observed before. Several corrections were tried for the small background signal, but fail to remove this feature from the spectrum.

We expect positronium in free spaces between the grains to decay in three ways: self-decay into 3 gammas, collisional pick-off annihilation, and collisional conversion followed by fast $p$-Ps decay. The first process gives no contribution in these $2 \gamma$-AMOC spectra. The two last processes contribute to the spectrum with their typical momentum, low for conversion and high for pick-off. To explain the observed change in $S$, the momentum distributions or the cross-section of the annihilation processes should change with age. Because the momentum contribution of the pick-off process is dominated by the momenta of the surface electrons, no change is expected in the momentum distribution of this process. For momentum distribution of the conversion process where positronium thermalisation could be seen, only a small opposite effect could be expected.

An age dependence of the cross-section is feasible when the cross-section depends on the kinetic energy of the Ps and slow thermalisation takes place.

An increase in the pick-off annihilation cross-section with energy is very unlikely. Lifetime experiments have shown that the $o$-Ps quenching rate is proportional with $\sqrt{T}$ in a wide temperature range, indicating no energy dependence.

The only explanation left is a strong decrease in the conversion cross-section with energy. Only for the conversion at gas molecules some data are available about the energy dependence of the conversion process. According to Charlton and Humberston [13], conversion quenching at paramagnetic atomic hydrogen can occur at all energies and no excitation of the atom needs to take places. Hara and Fraser [14] calculated this conversion cross-section for atomic hydrogen. Their results show only a minor increase in the cross-section at thermal energy. The conversion process with paramagnetic $\mathrm{O}_{2}$ gas has been studied experimentally by Kakimoto et al. $[15,16]$. Additionally to an elastic conversion process they found an inelastic conversion process. This inelastic process has an energy threshold of about $1 \mathrm{eV}$ and the cross-section was estimated to be at least two orders of magnitude higher than the elastic process. A similar process could explain the effects observed in our data. It would indicate a very low threshold energy because the change in cross-section happens at longer age where the positronium is almost thermalised.

\section{Conclusions}

We have shown that the use of age-momentum correlation experiments at a relativistic positron beam can give new information about the interaction of positronium with the grain surfaces of metal-oxide powders. We confirmed the creation of paramagnetic defect centres at low temperatures by positron and electron irradiation in the $\mathrm{Al}_{2} \mathrm{O}_{3}$ sample. A qualitative analysis of the observed spectra gives indication for a strong energy dependence of the conversion quenching 
cross-section. A more quantitative analysis should be made to confirm this hypothesis.

\section{Acknowledgments}

The authors would like to acknowledge the Max-Planck-Institut für Metallforschung in Stuttgart for the beam time at the pelletron. Especially we would like to thank Dr. H. Stoll for his cooperation and support.

\section{References}

[1] W.C. Sauder, J. Res. Natl. Bur. Stand. Sect. A 72, 91 (1968).

[2] T. Chang, M. Xu, X. Zeng, Phys. Lett. A 126, 189 (1987).

[3] Y. Nagashima, M. Kakimoto, T. Hyodo, K. Fujiwara, A. Ichimura, T. Chang, J. Deng, T. Akahane, T. Chiba, K. Suzuki, B.T.A. McKee, A.T. Stewart, Phys. Rev. A 52, 258 (1995).

[4] C. Dauwe, T. Van Hoecke, D. Segers, in: Proc. XXX Zakopane School of Physics, Eds. K. Tomala, E.A. Görlich, Institute of Physics, Jagiellonian University and H. Niewodniczański Institute of Nuclear Physics, Kraków 1995, p. 275.

[5] M. Skalsey, J.J. Engbrecht, R.K. Bithell, R.S. Vallery, D.W. Gidley, Phys. Rev. Lett. 80, 3727 (1998).

[6] S. Takada, T. Iwata, K. Kawashima, H. Saito, Y. Nagashima, T. Hyodo, Radiat. Phys. Chem. 58, 781 (2000).

[7] Mbungu-Tsumbu, C. Dauwe, M.D. Diatezwa, L. Mbosei, K. Motoko, Appl. Phys. A 48, 343 (1989).

[8] C. Dauwe, Mbungo-Tsumbu, Phys. Rev. B 45, 9 (1992).

[9] C. Dauwe, B. Van Waeyenberge, D. Segers, T. Van Hoecke, J. Kuriplach, J. Radioanal. Nucl. Chem. 210, 293 (1996).

[10] H. Saito, Y. Nagashima, T. Hyodo, T. Chang, Phys. Rev. B 52, 689 (1995).

[11] H. Saito, T. Hyodo, Phys. Rev. B 60, 11070 (1991).

[12] B. Van Waeyenberge, C. Dauwe, H. Stoll, Mater. Sci. Forum 363-365, 401 (2001).

[13] M. Charlton, J.W. Humberston, Positron Physics, Cambridge University Press, Cambridge 2001, p. 328.

[14] S. Hara, P.A. Fraser, J. Phys. B, At. Mol. Phys. 8, L472 (1975).

[15] M. Kakimoto, T. Hyodo, T. Chiba, T. Akahane, T.B. Chang, J. Phys. B, At. Mol. Phys. 20, L107 (1987).

[16] M. Kakimoto, T. Hyodo, T.B. Chang, J. Phys. B, At. Mol. Phys. 23, 589 (1990). 